\title{
Vascular Adventitia Calcification and Its Underlying Mechanism
}

\author{
$\mathrm{Na} \mathrm{Li}^{1}$, Wenli Cheng ${ }^{2}$, Tiequn Huang ${ }^{1}$, Jie Yuan ${ }^{3}, \mathrm{Xi} \mathrm{Wang}^{3}$, Meiyue Song ${ }^{3}$ \\ 1 Department of Health Care, China-Japan Friendship Hospital, Ministry of Health, Beijing, China, 2 Center \\ for Cardiovascular Diseases, China-Japan Friendship Hospital, Ministry of Health, Beijing, China, \\ 3 Graduate School, Beijing University of Traditional Chinese Medicine, Beijing, China \\ * chengwenli2013@163.com
}

\section{G openaccess}

Citation: Li N, Cheng W, Huang T, Yuan J, Wang X, Song M (2015) Vascular Adventitia Calcification and Its Underlying Mechanism. PLoS ONE 10(7): e0132506. doi:10.1371/journal.pone. 0132506

Editor: Francis Miller, Jr., University of lowa, UNITED STATES

Received: April 1, 2015

Accepted: June 15, 2015

Published: July 6, 2015

Copyright: $\odot 2015 \mathrm{Li}$ et al. This is an open access article distributed under the terms of the Creative

Commons Attribution License, which permits unrestricted use, distribution, and reproduction in any medium, provided the original author and source are credited.

Data Availability Statement: All relevant data are within the paper.

Funding: Supported by the general program of the national natural science foundation of China, No: 81173420. The funder has an role in study design, data collection and analysis, decision to publish, or preparation of the manuscript.

Competing Interests: The authors have declared that no competing interests exist.

\section{Abstract}

Previous research on vascular calcification has mainly focused on the vascular intima and media. However, we show here that vascular calcification may also occur in the adventitia. The purpose of this work is to help elucidate the pathogenic mechanisms underlying vascular calcification. The calcified lesions were examined by Von Kossa staining in ApoE -/- mice which were fed high fat diets (HFD) for 48 weeks and human subjects aged 60 years and older that had died of coronary heart disease, heart failure or acute renal failure. Explant cultured fibroblasts and smooth muscle cells (SMCs)were obtained from rat adventitia and media, respectively. After calcification induction, cells were collected for Alizarin Red S staining. Calcified lesions were observed in the aorta adventitia and coronary artery adventitia of ApoE-/-mice, as well as in the aorta adventitia of human subjects examined. Explant culture of fibroblasts, the primary cell type comprising the adventitia, was successfully induced for calcification after incubation with TGF- $\beta 1(20 \mathrm{ng} / \mathrm{ml})+$ mineralization media for 4 days, and the phenotype conversion vascular adventitia fibroblasts into myofibroblasts was identified. Culture of SMCs, which comprise only a small percentage of all cells in the adventitia, in calcifying medium for 14 days resulted in significant calcification.Vascular calcification can occur in the adventitia. Adventitia calcification may arise from the fibroblasts which were transformed into myofibroblasts or smooth muscle cells.

\section{Introduction}

Previously vascular calcification is considered passive and degenerative. Now it is recognized as a pathobiological process similar to embryonic bone formation. Vascular calcification is a common characteristic associated with atherosclerosis, hypertension, diabetes, vascular lesions, chronic kidney disease, and aging. Increased stiffness and decreased compliance in the vascular wall are the main consequences of vascular calcification, leading to myocardial ischemia, left ventricular hypertrophy, and heart failure, causing thrombosis and plaque rupture, these are part of pathological changes for myocardial ischemia/myocardial infarction. As a result, vascular calcification is thought to be one of the most important factors influencing cardiovascularand cerebrovascular-related diseases with high morbidity and high mortality rates [1-10]. 
Mechanical, inflammatory, metabolic, and morphogenetic signals might be involved in vascular calcification. The pathophysiology of vascular calcification is a complex process involving multiple cell types. Anatomically vascular calcification is typically divided into two categories: intimal atherosclerotic calcification and arterial medial calcification [11-12].

Previous research on vascular calcification has mainly focused on the arterial intima and media [1-2,12-13]. However, we show here that vascular calcification may also occur in the adventitia. So far, no one has reported adventitia calcification. In Timothy Ellam's study, we also find out the evidence of adventitia calcification in ApoE-/- mice (Fig 4c in reference 14) [14]. In Yu-Bin Sui's study, SD rats treated by intramuscular injections of vitamin D3 and feeding of nicotine also suffered from adventitia calcification (Fig 6Ae in reference 15)[15]. This finding increases the complexity of vascular calcification. Therefore, additional studies are required to elucidate the underlying pathogenic mechanisms governing vascular calcification.

\section{Materials and Methods}

\section{Animals and treatments}

Male Sprague-Dawley (SD) rats $(150 \pm 10 \mathrm{~g})(\mathrm{n}=3)$ and six-week-old male ApoE-deficient mice $(n=60)$ were obtained from the Animal Center, Peking University Health Science Center (Beijing). Animals were housed under standard conditions (room temperature $20 \pm 8^{\circ} \mathrm{C}$, humidity $60 \pm 10 \%$, lights from 6:00 to 18:00) and provided standard rodent chow and water freely.At 8 weeks of age, ApoE-deficient mice were switched to a high fat diet (HFD), consisting of $21 \%$ fat from lard and $1.25 \%$ (wt/wt) cholesterol for an additional 48 weeks.

\section{Tissue collection and processing}

ApoE-deficient mice were sacrificed after anesthetizing with sodium barbital. Hearts and aortas were harvested under sterile conditions. Aortas were fixed in 10\% formalin for histological and immunohistochemical observation. Aortic slices were collected consecutively from the beginning of the aortic valve to the point where the aortic valve disappeared (50 $\mu \mathrm{m}$ length and $5 \mu \mathrm{m}$ slice thickness). Aortas were also evaluated in a $3 \mathrm{~mm}$ section on the right side beneath the opening of the innominate artery. Bodies were stored at $4^{\circ} \mathrm{C}$, and autopsy was performed by the attending pathologist.

\section{Human samples}

Between December 1984 and January 2010, a total of 20 patients (14 males, 6 females) who were older than 60 years of age and who had died of coronary heart disease, heart failure (left ventricular ejection fraction $<45 \%$ ) or acute renal failure were enrolled in the study. They all had a history of coronary heart disease for more than 10 years, mean age 71.2 \pm 5 .9. Patients with chronic renal disease or hyperparathyroidism were excluded from the study. Patients were referred to the Department of Pathology of the China-Japan Friendship Hospital for autopsy. All procedures met the appropriate criteria for the use and handling of human tissue in China.

\section{Consent statement}

The study was approved by the Ethics committee of the China-Japan Friendship Hospital (No.2015-37) and was in accordance with the Helsinki Declaration. Written informed consents were obtained from all participants. All animals received humane care in compliance with the Animal Management Rule of the Ministry of Health, People's Republic of China 
(documentation no. 55, 2001) and the Care and Use of Laboratory Animals published by the US National Institutes of Health (NIH Publication No. 85-23, revised 1996).

\section{Materials}

Primary antibodies against vimentin, and all secondary antibodies, were purchased from Abcam (Cambridge, UK). Primary antibodies against smooth muscle (SM) $\alpha$-actin and $\beta$-Glycerophosphate and Alizarin Red S were from Sigma (St. Louis, MO, USA). Recombinant transforming growth factor-1 (TGF- $\beta 1$ ) was purchased from Pepro Tech Inc (Rocky Hill, NJ, USA). All chemicals and reagents were of analytical grade.

\section{Vascular smooth muscle cell (VSMC) culture in vitro}

Explant culture of VSMCs has been previously described [16]. Briefly, thoracic aortas from male SD rats were cut into small pieces after the removal of endothelium and adventitia. These sections were then placed in Dulbecco's modified Eagle's medium (DMEM) containing 20\% fetal bovine serum (FBS) and maintained at $37^{\circ} \mathrm{C}$ in an incubator containing $95 \%$ air and $5 \%$ $\mathrm{CO} 2$. VSMCs migrating from explants were collected and maintained in growth medium (DMEM containing 10\%FBS). Detection of $\alpha$-actin in cultured cells confirmed a positive response. VSMCs at passages five to eight were used for experiments.

\section{Fibroblast culture in vitro}

Explant culture of fibroblasts has been previously described [17]. Thoracic aorta adventitia from male SD rats were cut into small pieces after the removal of endothelium and media. These sections were placed in DMEM containing $20 \% \mathrm{FBS}$ and maintained at $37^{\circ} \mathrm{C}$ in an incubator containing $95 \%$ air and 5\%CO2. Fibroblasts migrating from explants were collected and maintained in growth medium (DMEM containing 10\%FBS). Detection of vimentin in cultured cells confirmed a positive response. The morphology and growth characteristics of the cells were typical of fibroblasts and were distinguished from smooth muscle cells by the absence of 'hill-and-valley' growth pattern and the lack of $\alpha$-actin staining at passages one. Fibroblasts at passages five to eight were used for experiments.

\section{Calcification of cells in vitro}

To induce calcification, confluent VSMCs were treated with $2.5 \mathrm{mmol} / \mathrm{L} \mathrm{CaCl}_{2}$ and $5 \mathrm{mmol} / \mathrm{L}$ $\beta$-glycerophosphate for 14 days.

To induce calcification, confluent fibroblasts were randomly divided into control group (DMEM containing $1 \% \mathrm{FBS}$ ), mineralization group (DMEM containing $1 \% \mathrm{FBS}, 50 \mathrm{ug} / \mathrm{ml}$ ascorbic acid, $5 \mathrm{mmol} / \mathrm{L} \beta$-glycerophosphate), TGF- $\beta 1$ ( $20 \mathrm{ng} / \mathrm{ml})+$ mineralization group (DMEM containing $1 \% \mathrm{FBS}, 50 \mathrm{ug} / \mathrm{ml}$ ascorbic acid, $5 \mathrm{mmol} / \mathrm{L} \beta$-glycerophosphate). The cells ( $\mathrm{n}=3$ wells/group) were treated for 4 days.

After calcification induction, cells were collected for Alizarin Red S staining or the detection of $\alpha$-actin. Each experiment was performed at least three times in triplicate.

\section{Alizarin Red S staining}

Alizarin Red S staining was performed as previously described with minor modification [16]. Cultured cells grown in 12-well plates were fixed in $4 \%$ formaldehyde in phosphate-buffered saline (PBS) for $45 \mathrm{~min}$ at $4^{\circ} \mathrm{C}$. Samples were, then washed in distilled water and exposed to Alizarin Red S (2\% aqueous, Sigma) for $5 \mathrm{~min}$. They were then washed again with distilled water and observed by microscopy. Positive staining is represented as a red/purple color. 


\section{Histological and immunohistochemical staining}

Paraffin sections were deparaffinized in xylene, dehydrated indecreasing alcohol solutions, and stained with Von Kossa for calcium deposition. For the detection of $\alpha$-actin (A2547, Sigma, USA), frozen slices of aorta were fixed in acetone and incubated with monoclonal antibody against $\alpha$-actin at a dilution of 1:200 at room temperature. For the detection of Vimentin (ab92547, Abcam, UK), fibroblasts were incubated with monoclonal antibody against Vimentin a dilution of 1:100. Cultured cells were incubated with monoclonal antibody against actin at a dilution of 1:200.

After washing, the slices were incubated in the dark with FITC-labeled secondary antibody at room temperature. Samples were then observed under a fluorescence microscope (Nikon 90i, Japan).

\section{Statistical analysis}

Statistical analysis involved use of SPSS v15(SPSS Inc., Chicago, IL, USA). Data are expressed as means \pm SME. Comparisons between two groups involvedt test. A twotailedP $<0.05$ was considered statistically significant.

\section{Results and Discussion}

\section{Calcification in the aorta adventitia of ApoE-/-mice}

After 48 weeks of being fed a HFD, significant calcified lesions were observed in the aorta adventitia of ApoE-/-mice. Calcified lesions were also detectable in the intima and media (Fig 1) and in the coronary artery adventitia and intima (Fig 2). Calcified areas were detectable by Von Kossa staining. Adventitia calcification was observed in 10 percent ApoE-/-mice samples.

\section{Calcification in the human aorta adventitia}

Adventitia calcification was observed in 50 percent human samples (Fig 3). The human were older than 60 years of age and had died of coronary heart disease, heart failure or acute renal failure. They all had a history of coronary heart disease for more than 10 years.

\section{VSMC calcification in vitro}

To reproduce the same effect in vitro, we established primary VSMCs in culture. After incubation with calcifying media for 14 days, significant calcification was detected in VSMCs by Alizarin Red S staining (Fig 4).

\section{Myofibroblasts calcification in vitro}

Successful in vitro culture of fibroblasts was confirmed by Vimentin staining. Fibroblasts were then incubated with control media for 4 days. Alizarin Red S staining of these cells was negative (Fig 5A). In contrast to VSMCs, calcification was not induced in the necrotic fibroblasts of mineralization group after incubation with mineralization media (DMEM containing 1\% FBS, $50 \mathrm{ug} / \mathrm{ml}$ ascorbic acid, $5 \mathrm{mmol} / \mathrm{L} \beta$-glycerophosphate) for 4 days. False positive was detected in fibroblasts by Alizarin Red S staining (Fig 5B). Calcification was detected in myofibroblasts of TGF- $\beta 1(20 \mathrm{ng} / \mathrm{ml})+$ mineralization group by Alizarin Red S staining (Fig 5C).

A-actin was stained positive in $9.3 \% \pm 1.5 \%$ of cells in control group (Fig 6A). False positive was detected in mineralization group (Fig 6B). A-actin positive was observed in $40.4 \% \pm 4.8 \%$ cells of TGF- $\beta 1(20 \mathrm{ng} / \mathrm{ml})+$ mineralization group (DMEM containing $1 \% \mathrm{FBS}, 50 \mathrm{ug} / \mathrm{ml}$ ascorbic acid, $5 \mathrm{mmol} / \mathrm{L} \beta$-glycerophosphate) $(\mathrm{P}<0.01)($ Fig $6 \mathrm{C})$. The phenotype conversion of 

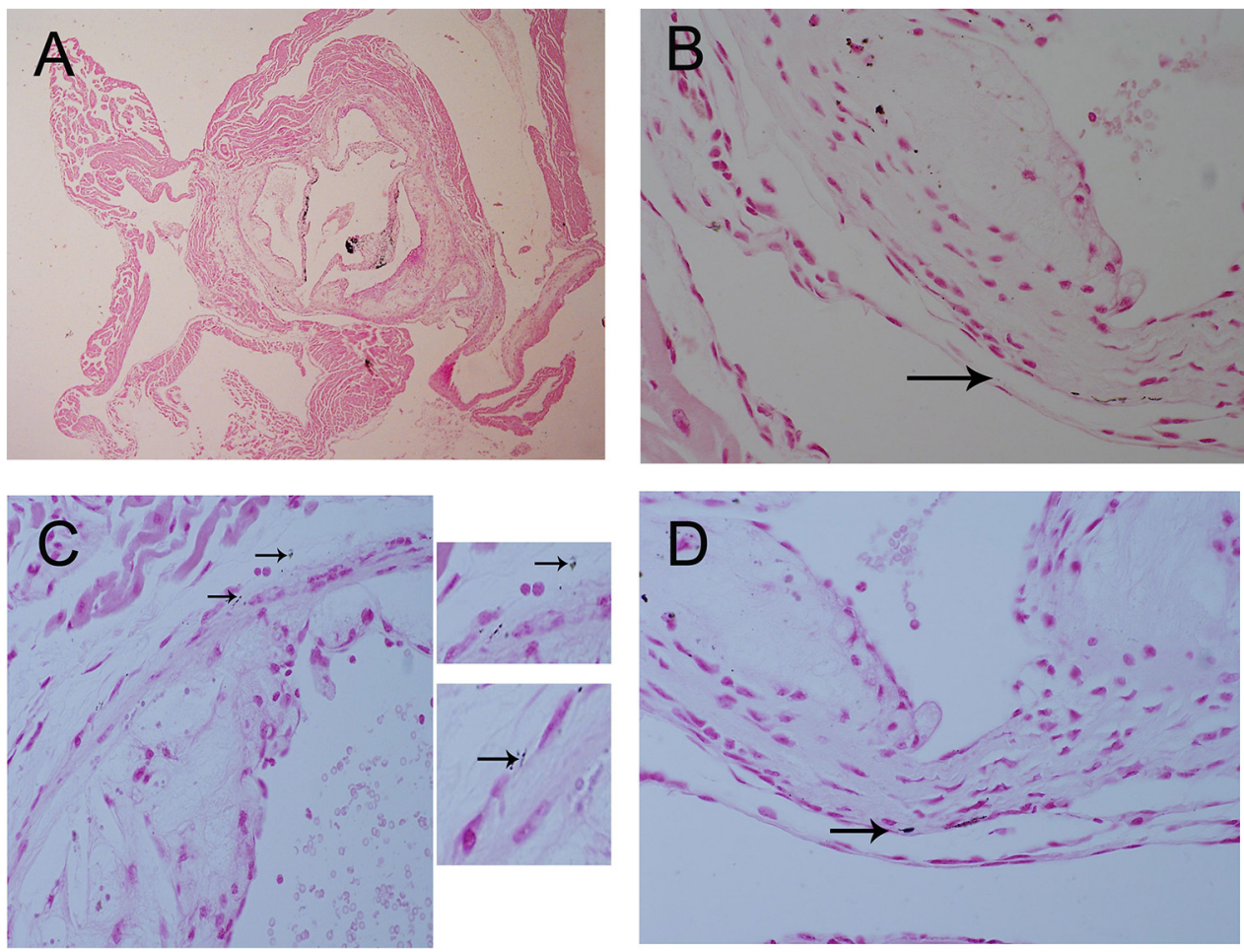

Fig 1. Calcification in the 56-week-old ApoE-/-mice aorta artery by Von Kossa staining. Von Kossa staining showing calcification in the 56-week-old ApoE-/-mice aorta adventitia, intima, and media. Magnification*40 (Fig 1A), *600 (Fig 1B-1D). Arrows indicate calcification in adventitia.

doi:10.1371/journal.pone.0132506.g001

vascular adventitial fibroblasts into myofibroblasts was identified. Quantification of $\alpha$-actin positive rate was performed by randomly taking five microscopic fields and the mean was calculated.

\section{A-actin expression}

$\alpha$-actin expression in the ApoE-/-mice aorta adventitia, media, and intima (Fig 7).
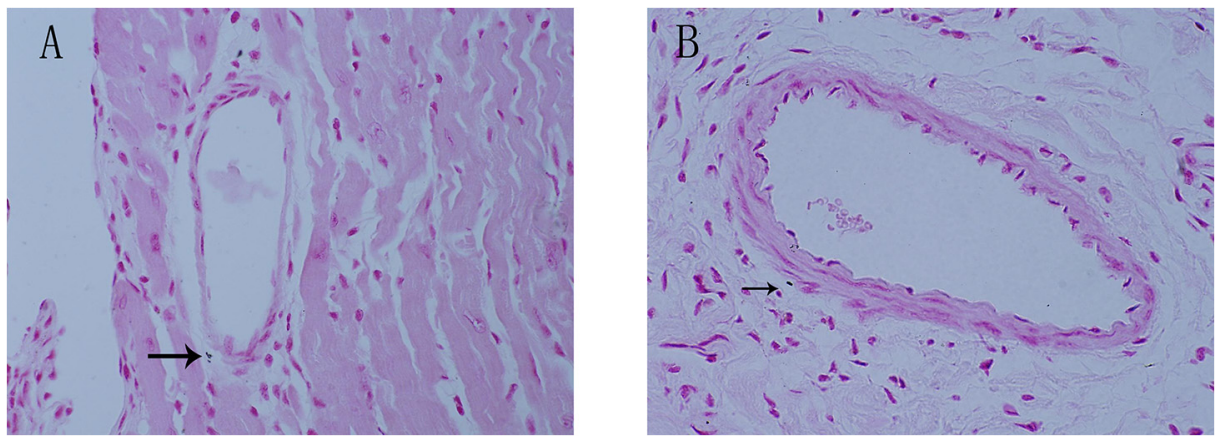

Fig 2. Calcification in the 56-week-old ApoE-/-mice coronary artery. Von Kossa staining showing calcification in the 56-week-old ApoE-/-mice coronary artery, adventitia, and intima. Magnification *600 (Fig 2A.2B). Arrows indicate calcification in adventitia.

doi:10.1371/journal.pone.0132506.g002 

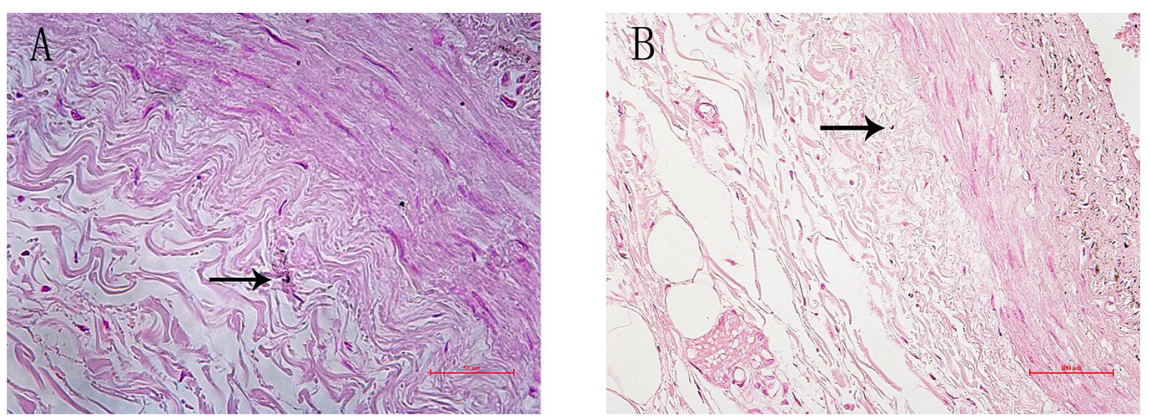

Fig 3. Calcification in the human thoracicaorta adventitia. Von Kossa staining showing calcification in the human thoracicaorta adventitia. The human were older than 60 years of age and had died of coronary heart disease, heart failure or acute renal failure. Arrows indicate calcification in adventitia.

doi:10.1371/journal.pone.0132506.g003

\section{Adventitia is involved in the formation and progression of atherosclerosis}

Traditionally, the primary function of adventitia has been thought to support vessels, provide nutrients for intima, and to maintain sympathetic nerve endings. However, a growing body of evidence indicates that adventitia also actively participates in atherosclerosis, and adventitia inflammation occurs in atherosclerotic disease [18]. Multiple inflammatory cell types, including macrophages, lymphocytes, dendritic cells, and mast cells have been found to infiltrate the adventitia and promote inflammation [19]. Adventitia inflammation in atherosclerotic arteries is widespread and proportional to the severity of atherosclerosis [20]. Importantly, adventitia inflammation can also spread to the intima, promoting development of atherosclerosis in this tissue as well [21].

\section{Adventitia calcification}

In this study, we examined adventitia calcification in atherosclerosis in ApoE-/- mice. Additionally, we found significant levels of calcification in the aorta adventitia of elderly patients. Our results also showed great difference in percentages of adventitia calcification between humans and ApoE-/-mice, which might be associated with the following reasons: 1) in the process that the mouse model of atherosclerosis results in calcification, there is only one single factor, while in the case of human factors are more complex; 2) the relatively smaller human

A

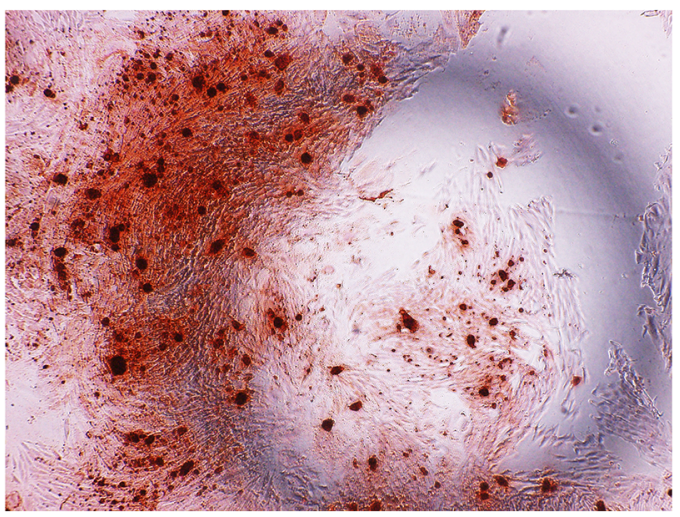

B

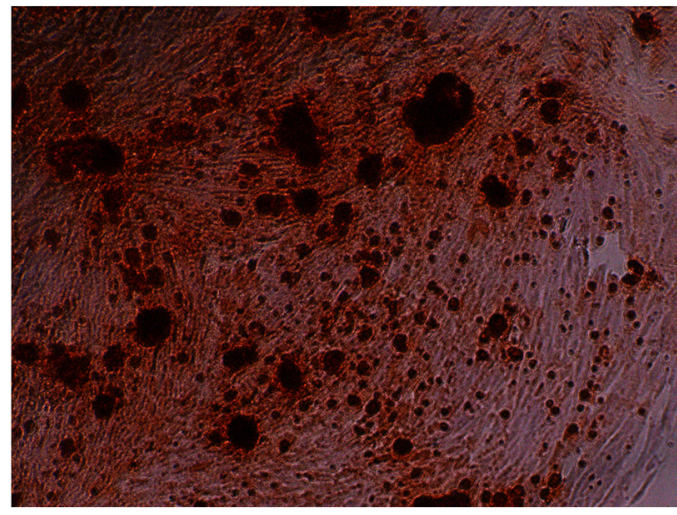

Fig 4. Alizarin Red S staining for VSMCs. Alizarin Red S staining for VSMCs on day 14. Magnification *40 (Fig 4A), ${ }^{*} 100$ (Fig 4B). 

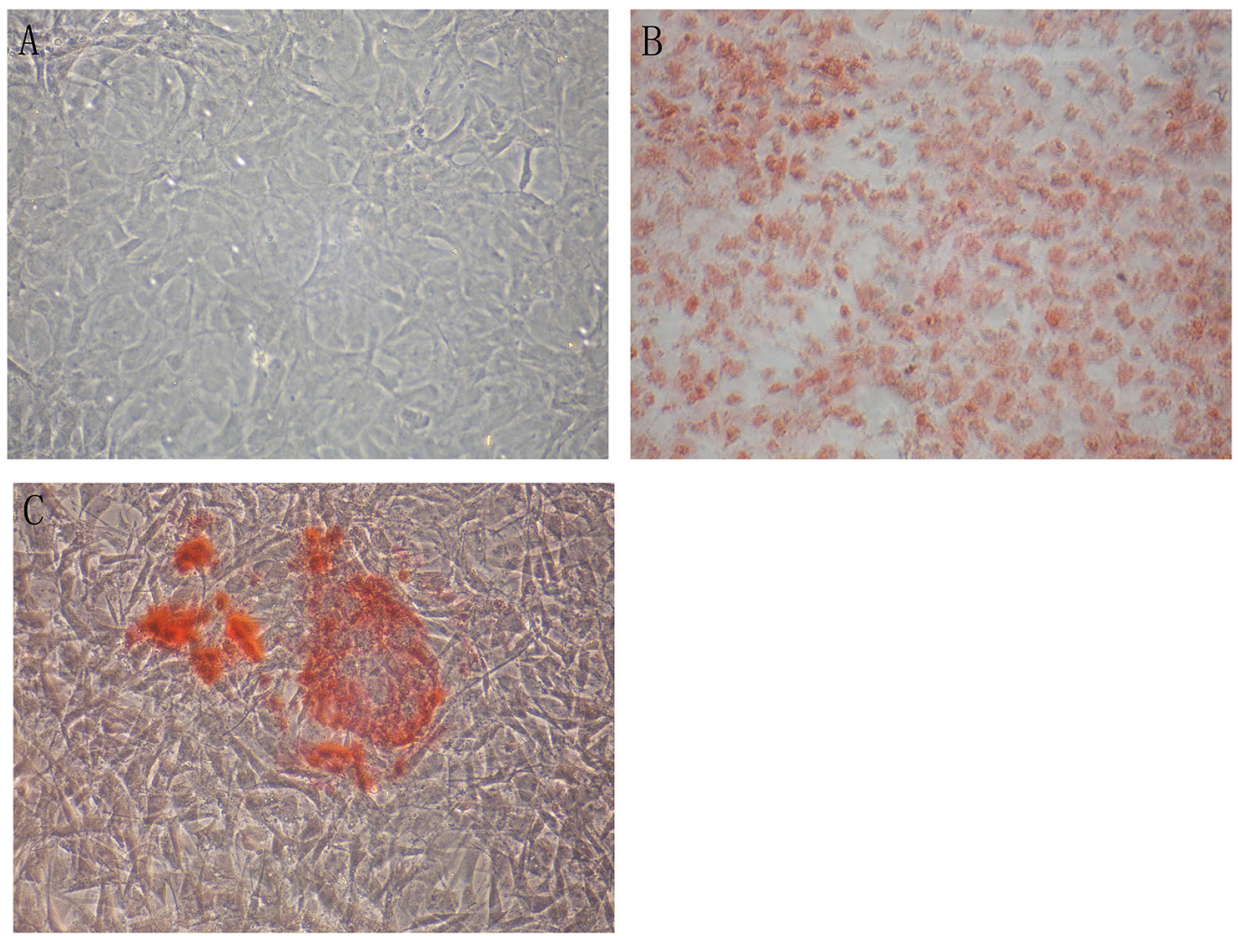

Fig 5. Alizarin Red S staining was positive for myofibroblasts. Fibroblasts were incubated with control media for 4 days. Alizarin Red S staining of these cells was negative(Fig 5A). Calcification was not induced in the necrotic fibroblasts of mineralization group after incubation with mineralization media (DMEM containing $1 \%$ FBS, $50 \mathrm{ug} / \mathrm{ml}$ ascorbic acid, $5 \mathrm{mmol} / \mathrm{L} \beta$-glycerophosphate) for 4 days. False positive was detected in fibroblasts by Alizarin Red S staining (Fig 5B). Calcification was detected in myofibroblasts of TGF- $\beta 1$ ( $20 \mathrm{ng} /$ $\mathrm{ml}$ ) + mineralization group by Alizarin Red S staining (Fig 5C). Expression of $\alpha$-actin in the myofibroblasts. Control group (Fig 6A), mineralization group (Fig 6B), TGF- $\beta 1(20 \mathrm{ng} / \mathrm{ml})+$ mineralization group (Fig 6C). Magnification *200

doi:10.1371/journal.pone.0132506.g005

sample size constrained by the experiment condition may be another reason; 3 ) there may be species difference in adventitia calcification.

However, the mechanisms governing adventitia calcification are not clear, and there have been no previous reports describing a relationship between adventitia inflammation and vascular adventitia calcification. Vascular media and intima calcification result, in part, from proinflammatory Ang II signaling cascades, that involve calpain-1, matrix metalloproteinases -2/9 (MMP-2/9), monocyte chemoattractant protein-1 (MCP-1), TGF- $\beta 1$ activation and milk fat globule epidermal growth factor-8 (MFG-E8) [22-26]. We propose that adventitia, under the pressure of inflammation and atherosclerosis, undergoes a series of complex changes that contribute to adventitia calcification.

\section{Cell types involved in adventitia calcification}

Adventitia is significantly comprised of collagen fiber and elastic fiber, fibroblast cells, and a small amount of smooth muscle cells. Vascular calcification is associated with several diseases, including hypertension, diabetes, chronic renal failure, and kidney dialysis. In addition, high calcium, high phosphorus, vitamin D3, and nicotine treatment have all been shown to induce vascular calcification in rats. The pathology of vascular calcification involves multiple cell types. For example, smooth muscle cells (SMCs) have been shown to the origin of osteochondroblastic cells [27]. 
A

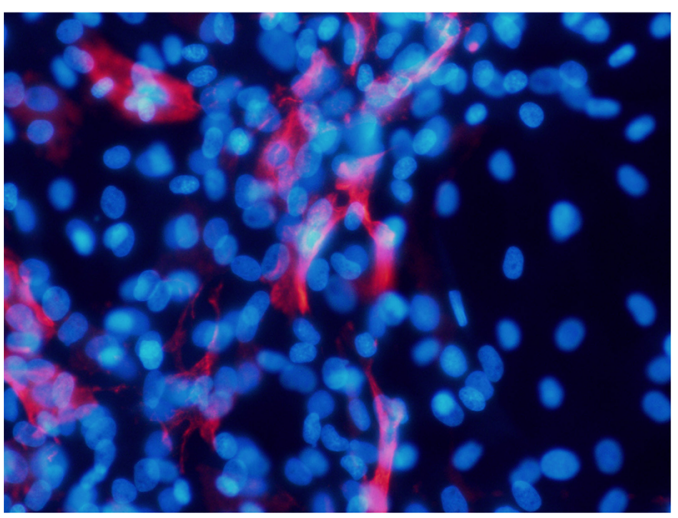

B

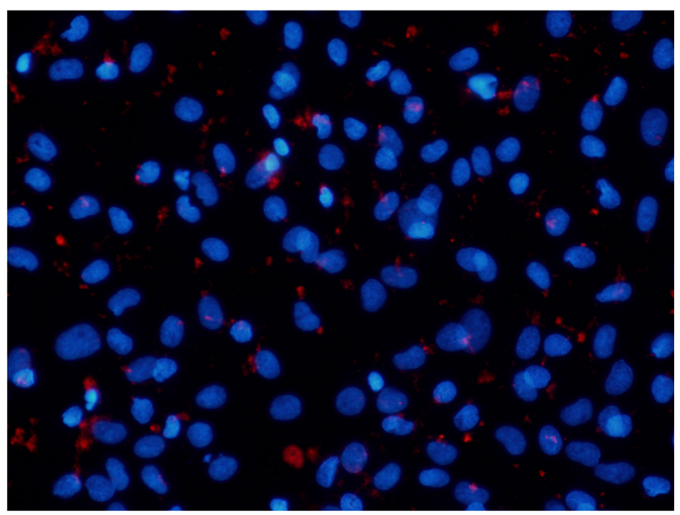

C

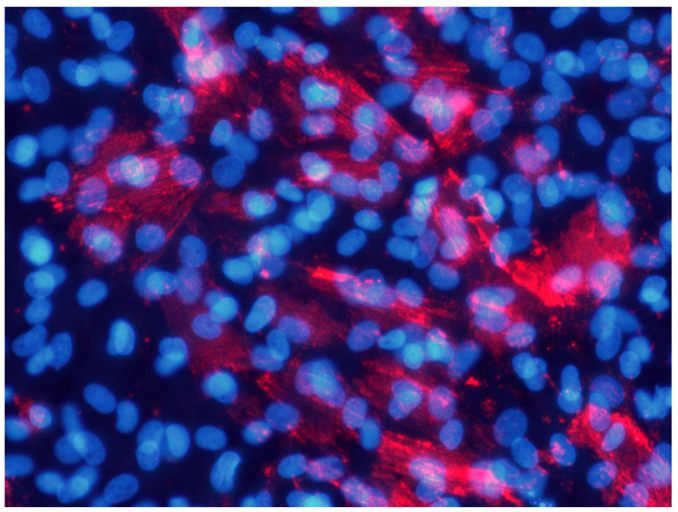

Fig 6. Alizarin Red S staining was positive for myofibroblasts. Fibroblasts were incubated with control media for 4 days. Alizarin Red S staining of these cells was negative (Fig $5 \mathrm{~A}$ ). Calcification was not induced in the necrotic fibroblasts of mineralization group after incubation with mineralization media (DMEM containing $1 \% \mathrm{FBS}, 50 \mathrm{ug} / \mathrm{ml}$ ascorbic acid, $5 \mathrm{mmol} / \mathrm{L} \beta$-glycerophosphate) for 4 days. False positive was detected in fibroblasts by Alizarin Red S staining (Fig 5B).Calcification was detected in myofibroblasts of TGF- $\beta 1(20 \mathrm{ng} / \mathrm{ml})+$ mineralization group by Alizarin Red S staining (Fig 5C). Expression of $\alpha-a c t i n$ in the myofibroblasts. Control group (Fig 6A), mineralization group (Fig 6B), TGF- $\beta 1(20 \mathrm{ng} / \mathrm{ml})+$ mineralization group (Fig 6C). Magnification *200

doi:10.1371/journal.pone.0132506.g006

Calcifying vascular cells (CVCs) primarily exist in the vascular wall of the intima. CVCs have characteristics of osteogenic cells, expressing alkaline phosphatase and secreting collagen I, osteopontin (OPN), osteocalcin, and osteonectin. CVCs also display some mesenchymal cell characteristics, which may help promote the trans-differentiation process [28].

Pericytes are star-shaped, multi-functional cells that are found throughout the large, medium, and small artery intima in the inner layer, media outer layer, and the outer nourishing blood vessels. Pericytes can differentiate into macrophages, fat cells, cartilage cells, and smooth muscle cells. Pericytes can also secrete extracellular matrix and form a large number of calcified nodules. They may secrete collagen I, OPN, and osteocalcin, which promote differentiation to osteogenic cells, bone cells, and bone tissue [28].

Mesenchymal stem cells, located in vessel walls, also contribute to vascular calcification. This is primarily due to their trans-differentiation to osteogenic cells when exposed to various calcifying factors [29]. 


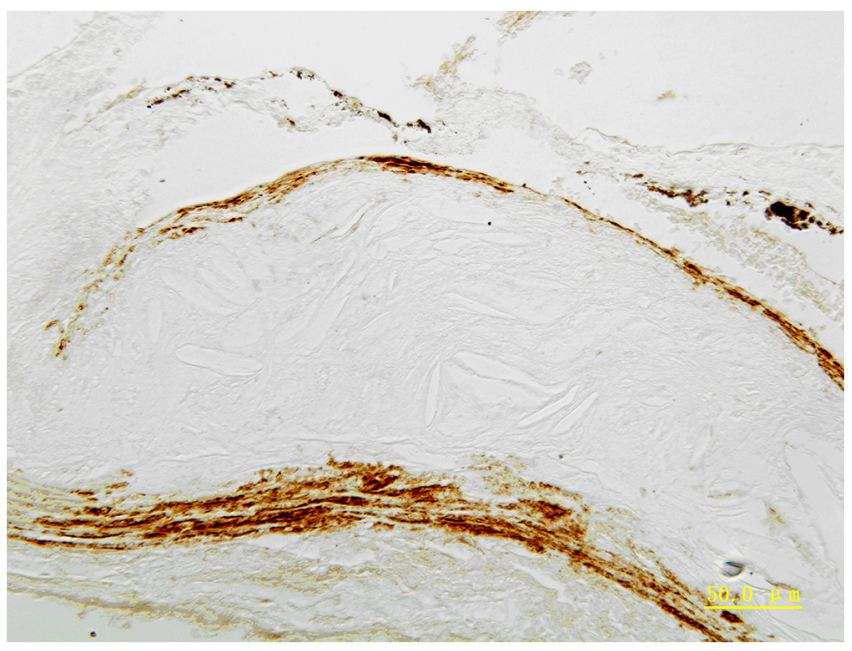

Fig 7. Expression of a-actin in the ApoE-/-mice aorta. Expression of a-actin in the ApoE-/-mice aorta adventitia, media, and intima. Magnification *400.

doi:10.1371/journal.pone.0132506.g007

Fibroblasts are the major cell type comprising the adventitia, and they are activated in the pathological state. Fibroblasts undergo phenotype transformation, proliferation, and migration. They can also secrete various cytokines, participating in the development of several diseases, including atherosclerosis. Physical damage, hypoxia, and inflammation can cause adventitia fibroblasts to differentiate into myofibroblasts, and this is mainly due to the actions of TGF-1. Myofibroblasts then express smooth muscle actin and collagen. Myofibroblasts contain stress fibers and cytoskeletal proteins with systolic and diastolic function. They can migrate, proliferate, and participate in the formation of the new intima. Adventitia fibroblast migration to the intima is the main cause of new intima thickening and luminal stenosis [30]. In addition, myofibroblasts can secrete a variety of cytokines and growth factors, including TGF-ß1, MCP-1, interleukin (IL), endothelin-1 (ET-1), tumor necrosis factor $\alpha$ (TNF- $\alpha$ ), MMPs, and HADPH oxidase, which contribute to adventitia inflammation. For example, TGF- $\$ 1$ enhances fibroblast phenotype transformation, and MCP-1 promotes the recruitment and activation of macrophages. ET-1 increases the deposition of extracellular matrix components [31]. Together, these factors promote inflammation. Finally, adventitia fibroblasts, in both physiological and pathological settings, produce reactive oxygen species(ROS), which is involved in oxidative stress.ROS production by adventitia fibroblasts has been shown to be important for cell proliferation and intima regeneration [32-34].

In this study, we show that fibroblasts, the primary cell type comprising the adventitia, succeeded in inducing calcification after incubation with TGF- $\beta 1(20 \mathrm{ng} / \mathrm{ml})+$ mineralization media for 4 days, and identified the phenotype conversion of vascular adventitial fibroblasts into myofibroblasts. Zhang et al. reported the phenotypic differentiation of adventitial fibroblasts into myofibrobalsts from rat aorta [35]. Lai et al. reported that mouse aortic myofibroblasts could induce calcification [17]. Additionally, Simionescu et al. reported that myofibroblasts derived from rat dermal fibroblasts could also induce calcification [36].

Vascular smooth muscle cells (VSMCs) are the primary cell type in the medium, but comprise only a small percentage of cell types found in the adventitia. VSMCs generate and secrete extracellular matrix components and likely contribute to the process of inflammation [37]. VSMCs also have the ability to contract, synthesis, proliferation and secrete multiple factors. The contractile VSMCs, co-exist with pools of proliferative, synthetic, or phagocytic VSMCs, 
drive the adaptation of vascular function [38]. The contractile VSMCs exists to maintain aortic wall elasticity. Upon mechanical stimulation or molecular signals, transformation of cell phenotype may occur [39]. Transforming the contractile VSMCs into synthetic VSMCs decreases the elasticity of the ascending aorta, and increased collagen deposition hardens the aortic tissue [39].A-SMA/SM22 $\alpha$ is a marker of contractile VSMCs in normal, healthy adult tissue. Expression of these markers is significantly decreased in embryonic vascular development or vessel damage repair. OPN is an additional marker that is often used. Upon transforming into synthetic VSMCs, OPN expression increases. In contrast, transforming into contractile VSMCs promotes a decrease in OPN expression.

Here, we show that in vitro culture of SMCs with calcification-inducing medium results in strong calcification of these cells. We propose that adventitia calcification also arises from SMCs, which comprise a small percentage of the adventitia cell population. We detected positive expression of a-actin in the aorta adventitia, media, and intima of ApoE-/-mice. These $\alpha$ actin positive cells in the adventitia could be SMCs but could also be myofibroblasts. The adventitia is mainly comprised of the fibroblasts, as well as tiny amount of smooth muscle cells. Adventitia calcification may arise from the fibroblasts which were transformed into myofibroblasts or smooth muscle cells.

Our subjects only involved the large arteries and did not include the medium and small arteries, which is our limitation of the study.

Recent reports have described other mechanisms that may contribute to the process, including mechanical stimulation, biochemical factors, and micro-RNA regulation. Additional studies are needed to fully understand the process of vascular calcification and cell phenotype transformation. Such studies may help clinical treatment in the future.

\section{Conclusions}

Vascular calcification can occur in the adventitia. The adventitia is mainly comprised of the fibroblasts, as well as tiny amount of smooth muscle cells. Adventitia calcification may arise from the fibroblasts which were transformed into myofibroblasts or smooth muscle cells.

\section{Acknowledgments}

We are greatly indebted to Dr.Mingyi Wang, Mr.Jing Zhang and Dr. Yongfen Qi for the expert technical assistance.

\section{Author Contributions}

Conceived and designed the experiments: NL WC. Performed the experiments: NL TH JY XW MS. Analyzed the data: NL WC. Contributed reagents/materials/analysis tools: NL WC TH. Wrote the paper: NL WC.

\section{References}

1. Johnson RC, Leopold JA, Loscalzo J. Vascular calcification: pathobiological mechanisms and clinical implications. Circ Res. 2006; 99:1044-1059. PMID: 17095733

2. Cianciolo G, Donati G, La Manna G, Ferri A, Cuna V, Ubaldi G, et al.The cardiovascular burden of endstage renal disease patients. Minerva Urol Nefrol.2010; 62:51-66. PMID: 20424570

3. Newman AB, Naydeck BL, Sutton-Tyrrell K, Feldman A, Edmundowicz D, Kuller LH.Coronary artery calcification in older adults to age 99:prevalence and risk factors. Circulation. 2001; 104:2679-2684. PMID: 11723018

4. Moe SM, O'Neill KD, Reslerova M, Fineberg N, Persohn S, Meyer CA. Natural historyof vascular calcification in dialysis and transplant patients.Nephrol Dial Transplant. 2004; 19:2387-2393. PMID: 15252163 
5. Ohtsuka S, Kakihana M, Watanabe H, Sugishita Y.Chronically decreased aortic distensibility causes deterioration of coronary perfusion during increased left ventricularcontraction. J Am Coll Cardiol 1994; 24:1406-1414. PMID: 7930267

6. Iribarren C, Sidney S, Sternfeld B, Browner WS.Calcification of the aortic arch: risk factors and associationwith coronary heart disease, stroke and peripheral vasculardisease. JAMA 2000; 283:2810-2805. PMID: 10838649

7. Mitchell JR, Adams JH. Aortic size and aortic calcification.A necropsy study. Atherosclerosis 1977; 27:437-446. PMID: 884000

8. Dart AM, Lacombe F, Yeoh JK, Cameron JD, Jennings GL, Laufer E, et al. Aortic distensibility inpatients with isolated hypercholesterolaemia, coronary arterydisease, or cardiac transplant. Lancet 1991; 338:270-273. PMID: 1677109

9. Simonson E, Nakagawa K. Effect of age on pulse wavevelocity and "aortic ejection time" in healthy men and in menwith coronary artery disease. Circulation 1960; 22:126-129. PMID: 14446988

10. Harvey A, Montezano AC, Touyz RM. Vascular biology of ageing-Implications in hypertension.J Mo Cell Cardiol. 2015 Apr 17. pii: S0022-2828(15)00126-1. doi: 10.1016/j.yjmcc.2015.04.011. [Epub ahead of print].

11. Demer LL, Tintut Y.Vascular calcification: pathobiology of a multifaceted disease.Circulation.2008; 117:2938-2948. doi: 10.1161/CIRCULATIONAHA.107.743161 PMID: 18519861

12. Lanzer Peter, Boehm Manfred, Sorribas Victor, Thiriet Marc, Janzen Jan,Zeller Thomas, et al.Medial vascular calcification revisited: review and perspectives. European Heart Journal.2014; 35: 15151525. doi: 10.1093/eurheartj/ehu163 PMID: 24740885

13. Persy V, D'Haese P.Vascular calcification and bone disease: the calcification paradox. Trends Mol Med.2009; 15:405-416. doi: 10.1016/j.molmed.2009.07.001 PMID: 19733120

14. Ellam Timothy, Hameed Abdul, Haque Risat ul, Muthana Munitta, Wilkie Martin, Francis Sheila E., et al Vitamin D Deficiency and Exogenous Vitamin D ExcessSimilarly Increase Diffuse Atherosclerotic Calcification inApolipoprotein E Knockout Mice.PloS ONE.2014 Feb 19; 9(2). doi: 10.1371/journal.pone. 0088767

15. Sui Yu-Bin, Chang Jin-Rui, Chen Wen-Jia, Zhao Lei, Zhang Bao-Hong,Yu Yan-Rong, et al.Angiotensin-(1-7) inhibits vascular calcification in rats. Peptides.2013; 42: 25-34. doi: 10.1016/j. peptides.2012. 12.023 PMID: 23291307

16. Cai Y, Xu MJ, Teng X, Zhou YB, Chen L, Zhu Y, et al. Intermedin inhibits vascular calcification by increasing the level of matrix gamma-carboxyglutamic acid protein. Cardiovasc Res.2010, 85:864873. doi: 10.1093/cvr/cvp366 PMID: 19910445

17. Lai CF, Shao JS, Behrmann A, Krchma K, Cheng SL,Towler DA.TNFR1-activatedreactive oxidative species signals up-regulate osteogenic Msx2 programs in aortic myofibroblasts. Endocrinology. 2012; 153:3897-3910. doi: 10.1210/en.2012-1216 PMID: 22685265

18. Sakamoto Sumiharu,Tsuruda Toshihiro,Hatakeyama Kinta,Imamura Takuroh,Asada Yujiro, Kitamura K. Impact of Age-Dependent Adventitia Inflammation on Structural Alteration of Abdominal Aorta in Hyperlipidemic Mice. PloS ONE.2014 Aug 25; 9(8). doi: 10.1371/journal.pone.0105739

19. Rayner K, Van Eersel S, Groot PH, Reape TJ.Localisation of mRNA for JE/MCP-1 and its receptor CCR2 in atherosclerotic lesions of the ApoE knockout mouse.J Vasc Res. 2000; 37:93-102. PMID: 10754394

20. Moreno PR, Purushothaman KR, Fuster V, O'Connor WN. Intimomedial interface damage and adventitial inflammation is increased beneath disrupted atherosclerosis in the aorta: implications for plaque vulnerability. Circulation. 2002; 105:2504-2511. PMID: 12034657

21. Wilcox JN, Scott NA.Potential role of the adventitia in arteritis and atherosclerosis.Int J Cardiol. 1996; 54 Suppl:S21-35. PMID: 9119525

22. Wang $M$, Khazan B, Lakatta EG. Central Arterial Aging and Angiotensin II Signaling. Curr Hypertens Rev. 2010; 6:266-281. PMID: 21423831

23. Jiang L, Zhang J, Monticone RE, Telljohann R, Wu J, Wang M, et al. Calpain-1 regulation of matrix metalloproteinase 2 activity in vascular smooth muscle cells facilitates age-associated aortic wall calcification and fibrosis. Hypertension. 2012; 60:1192-1199. doi: 10.1161/HYPERTENSIONAHA.112. 196840 PMID: 23006733

24. Yan J, Stringer SE, Hamilton A, Charlton-Menys V, Götting C, Müller B, et al.Decorin GAG synthesis and TGF- $\beta$ signaling mediate $O x-L D L$-induced mineralization of human vascular smooth muscle cells. Arterioscler Thromb Vasc Biol. 2011; 31:608-615. doi: 10.1161/ATVBAHA.110.220749 PMID: 21205989

25. Nesti LJ, Caterson EJ, Li WJ, Chang R, McCann TD, Hoek JB, et al. TGF-beta1 calcium signaling in osteoblasts. J Cell Biochem. 2007; 101:348-359. PMID: 17211850 
26. Wang M, Zhao D, Spinetti G, Zhang J, Jiang LQ, Pintus G, et al. Matrixmetalloproteinase 2 activation of transforming growth factor-beta1 (TGF-beta1) and TGF-beta1-type II receptor signaling within the aged arterial wall. Arterioscler Thromb Vasc Biol. 2006; 26:1503-1509. PMID: 16690877

27. Hruska KA.Vascular smooth muscle cells in the pathogenesis of vascular calcification.Circ Res.2009, 104:710-711. doi: 10.1161/CIRCRESAHA.109.195487 PMID: 19325156

28. Shao JS, Cai J, Towler DA.Molecular mechanisms of vascular calcification: lessons learned from the aorta. Arterioscler ThrombVasc Biol.2006; 26:1423-1430.

29. Sata M.Circulating vascular progenitor cells contribute to vascular repair, remodeling, and lesion formation.Trends Cardiovasc Med. 2003; 13:249-53. PMID: 12922022

30. Dunkern TR, Feurstein D, Rossi GA, Sabatini F, HatzelmannA. Inhibition of TGF-beta induced lung fibroblast to myofibroblast conversion by phosphodiesterase inhibiting drugs and activators of soluble guanylylcyclase. Eur J Pharmacol. 2007; 572:12-22. PMID: 17659276

31. Maiellaro K, Taylor WR.The role of the adventitia in vascular inflammation.Cardiovasc Res.2007; 75:640-648. PMID: 17662969

32. Di Wang H, Hope S, Du Y, Quinn MT, Cayatte A, Pagano PJ, et al. Paracrine role of adventitial superoxide anion in mediating spontaneous tone of the isolated rat aorta in angiotensin II-induced hypertension.Hypertension.1999; 33:1225-1232. PMID: 10334816

33. Dourron HM, Jacobson GM, Park JL, Liu J, Reddy DJ, Scheel ML, et al.Perivascular gene transfer of NADPH oxidase inhibitor suppresses angioplasty-induced neointimal proliferation of rat carotid artery. Am J Physiol Heart Circ Physiol. 2005; 288:H946-953. PMID: 15388496

34. Weaver M, Liu J, Pimentel D, Reddy DJ, Harding P,Peterson EL,et al.Adventitial delivery of dominantnegative p67phox attenuates neointimal hyperplasia of the rat carotid artery. Am J Physiol Heart Circ Physiol.2006; 290:H1933-1941. PMID: 16603705

35. Zhang YG, Hu YC, Mao YY, Wei RH, Bao SL, Wu LB, et al. Transforming growth factor- $\beta 1$ involved in urotensin II-induced phenotypic differentiation of adventitial fibroblasts from rat aorta.Chin Med J.2010; 123:3634-3639. PMID: 22166643

36. Simionescu A, Simionescu DT, Vyavahare NR. Osteogenic responses in fibroblasts activated by elastin degradation products and transforming growth factor-beta1: role of myofibroblasts in vascular calcification.Am J Pathol. 2007; 171:116-123. PMID: 17591959

37. Bulick AS, Mufioz-Pinto DJ, Qu X. Impact of endothelial cell and nmchanical conditioning on smooth muscle cell extraeellular matrix uroduction and differentiation. Tissue Eng Part A,2009; 15:815-825. doi: 10.1089/ten.tea.2008.0179 PMID: 19108675

38. Lacolley P, Regnault V, Nicoletti A, Li Z,Michel JB. The vascular smooth muscle cell in arterial pathology: a cell that can take on multiple roles. Cardiovasc Res.2012; 95:194-204. doi: 10.1093/cvr/cvs135 PMID: 22467316

39. El-Hamamsy I, Yacoub MH. Cellular and molecular mechanisms of thoracic aortic aneurysms. Nat Rev Cardiol.2009; 6:771-786. doi: 10.1038/nrcardio.2009.191 PMID: 19884902 